\title{
Coexistence of Drift Waves and D'Angelo Modes at Different Position and Frequency in Linear Plasma Device
}

\author{
Nathan DUPERTUIS ${ }^{1)}$, Shigeru INAGAKI ${ }^{2,3)}$, Yoshihiko NAGASHIMA ${ }^{2,3)}$, Yusuke KOSUGA ${ }^{2,3)}$, \\ Fumiyoshi KIN $^{5)}$, Tatsuya KOBAYASHI ${ }^{4)}$, Naohiro KASUYA ${ }^{2,3)}$, Makoto SASAKI ${ }^{2,3)}$, \\ Akihide FUJISAWA ${ }^{2,3)}$, Minh Quang TRAN ${ }^{1)}$, Sanae-I. ITOH ${ }^{2,3)}$ and Kimitaka ITOH ${ }^{3,4)}$ \\ ${ }^{1)}$ Swiss Plasma Center, École Polytechnique Fédérale de Lausanne, CH-1015, Lausanne, Switzerland \\ ${ }^{2)}$ Research Institute for Applied Mechanics, Kyushu University, Kasuga 816-8580, Japan \\ ${ }^{3)}$ Research Center for Plasma Turbulence, Kyushu University, Kasuga 816-8580, Japan \\ ${ }^{4)}$ National Institute for Fusion Science, Toki 509-5292, Japan \\ ${ }^{5)}$ Interdisciplinary Graduate School of Engineering Science, Kyushu University, Kasuga 816-8580, Japan
}

(Received 23 September 2016 / Accepted 28 December 2016)

\begin{abstract}
Experimental plasma discharges in linear PANTA device are studied by Mach probe measurements, providing floating potential, ion saturation current, and parallel flow velocity time evolution, at different radii of the device. Spectral analysis indicates that drift waves and D'Angelo modes exist simultaneously in the plasma. A discrimination study shows they are located at different positions in radius and frequency.
\end{abstract}

(c) 2017 The Japan Society of Plasma Science and Nuclear Fusion Research

Keywords: drift wave, D’Angelo mode, coexistence, linear plasma device

DOI: $10.1585 / \mathrm{pfr} .12 .1201008$

Plasma turbulence is one of the central issues of modern plasma research. Extensive studies have been conducted and it is argued that the family of drift wave (DW) turbulence, which is driven by scalar gradients such as density, temperature, and pressure, plays an important role to understand confinement of fusion plasmas [1]. In addition to this, plasmas also support flows, which give rise to instability driven by vector fields [3,4]. For instance, Kelvin-Helmholtz instability [2] and/or D'Angelo mode (D'AM) [3-6] can arise from the inhomogeneity of the velocity in the perpendicular and the parallel direction to the magnetic fields, respectively, and impact the evolution of the system [7]. Since plasmas have several gradients, of both scalar and vector fields, it is likely that different types of modes/instabilities coexist and interact. As DWs and D'AMs play different roles on transport and structural formation [4], it is important to clarify the mechanism how/where these fluctuations are excited in plasmas. In this paper, we report an experimental observation of the simultaneous excitation of DWs driven by the density gradient and D'AMs driven by the parallel velocity gradients. Fluctuations are directly measured and power spectra are used to differentiate the modes.

Fluctuation of parallel velocity is a key quantity to discriminate DWs and D'AMs (see Table 1). It is well known that parallel velocity perturbations are weak in the case of DW, with respect to density perturbations [1]:

$$
\left|\frac{\tilde{v}_{\|}}{c_{s}}\right|^{2}=\left(\frac{k_{\|} c_{s}}{\omega_{* e}}\right)^{2}\left|\frac{\tilde{n}}{n_{0}}\right|^{2}<\left|\frac{\tilde{n}}{n_{0}}\right|^{2} .
$$

author'se-mail: nathan.dupertuis@alumni.epfl.ch
Table 1 Discriminant signature for drift waves and D'Angelo modes.

\begin{tabular}{|c|c|c|}
\hline Phenomenon & Power & Phase $\left(\frac{\tilde{v}_{\|}}{c_{s}}\right.$ w.r. $\left.\frac{\tilde{n}}{n_{0}}\right)$ \\
\hline \hline DW & $\left|\tilde{n} / n_{0}\right|^{2}>\left|\tilde{v}_{\|} / c_{s}\right|^{2}$ & small $(<1 \mathrm{rad})$ \\
\hline D'AM & $\left|\tilde{n} / n_{0}\right|^{2} \leq\left|\tilde{v}_{\|} / c_{s}\right|^{2}$ & high $(>1 \mathrm{rad})$ \\
\hline
\end{tabular}

In the case of D'AM $[5,6]$, the relation becomes:

$$
\left|\frac{\tilde{v}_{\|}}{c_{s}}\right|^{2}=\left(1+k_{\perp}^{2} \rho_{s}^{2}\right)\left(\frac{k_{y} \rho_{s}}{k_{\|} c_{s}}\left\langle v_{\|}\right\rangle^{\prime}-1\right)\left|\frac{\tilde{n}}{n_{0}}\right|^{2} .
$$

For most unstable mode $k_{\|} c_{s}=k_{y} \rho_{s}\left\langle v_{\|}\right\rangle^{\prime} / 2$. Then the relation reduces to

$$
\left|\frac{\tilde{v}_{\|}}{c_{s}}\right|^{2}=\left(1+k_{\perp}^{2} \rho_{s}^{2}\right)\left|\frac{\tilde{n}}{n_{0}}\right|^{2} .
$$

The coefficient between the normalized amplitudes is order of unity, slightly higher than 1 , so we get $\left|\tilde{v}_{\|} / c_{s}\right|^{2} \geq\left|\tilde{n} / n_{0}\right|^{2}$. The difference can be used to discriminate the modes. We note that, while the relation is derived from the linear response, a similar relation holds even for the nonlinear state, as indicated by numerical simulation [8].

An experiment was conducted to determine if and how DWs and D'AMs could coexist in linear plasma devices. Several plasma discharges were realized in PANTA device in Kyushu University [9], with Argon gas pressure of $3.0 \mathrm{~m}$ Torr and $B=0.09 \mathrm{~T}$, at parallel position $z=1625 \mathrm{~mm}$. $I_{i s}$ 
and $v_{\|}$are measured using Mach probes [10] at different radial positions from the device axis ( $r=20$ to $60 \mathrm{~mm}$, with $5 \mathrm{~mm}$ intervals). Then $v_{\|}$was computed from the two tips measurements for $I_{i s}$. Normalized power for $I_{i s}$ fluctuations is equivalent to normalized density fluctuations power: $\left|\widetilde{I_{i s}} /\left\langle I_{i s}\right\rangle\right|^{2}=\left|\tilde{n} / n_{0}\right|^{2}$, thus the discrimination study can be operated.

Radial profiles of $I_{i s}$ and $v_{\|}$are presented in Fig. 1 (a). The velocity gradient is higher around $r=30 \mathrm{~mm}$, thus this radius is likely to be more favourable for D'AM generation. Auto-power spectra for $I_{i s}$ and $v_{\|}$are shown at Mach probe radial position $r=30 \mathrm{~mm}$ in Fig. 1 (b) and $r=40 \mathrm{~mm}, r=50 \mathrm{~mm}$ in Figs. 2 (a) (b). At all radii, noise level is far lower than power spectrum level in the region of interest $(<10 \mathrm{kHz})$. For instance, noise levels for $I_{i s}$ and $v_{\|}$at $r=30 \mathrm{~mm}$ are respectively around $2.2 \cdot 10^{-10} \mathrm{~Hz}^{-1}$ and $1.1 \cdot 10^{-9} \mathrm{~Hz}^{-1}$.

All spectra for $I_{i s}$ show peaks at $1.1,2.2$, and $3.3 \mathrm{kHz}$, that are likely to be attributed to DWs as the normalized $I_{i s}$ power is higher than normalized $v_{\|}$power. Cross-phase study between the two measurements confirms that the phase is lower than $1 \mathrm{rad}$ for these frequencies. This is valid at all radii between 20 and $60 \mathrm{~mm}$.

However, at frequencies higher than $3.5 \mathrm{kHz}$, the figure shows different behaviour at $r=30 \mathrm{~mm}$ (and at some degree at $50 \mathrm{~mm}$ ). $\quad v_{\|}$spectrum overcomes $I_{i s}$ spectrum, about one order of magnitude higher at $10 \mathrm{kHz}$. Crossphase study between the two measurements also show
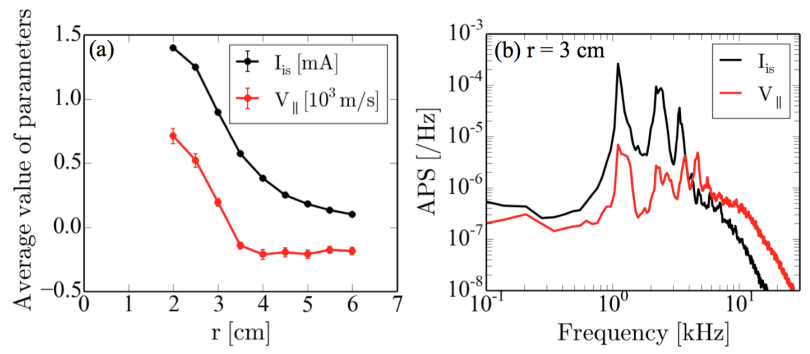

Fig. 1 Average value of parameters at different radii (a) and auto-power spectra for normalized ion saturation current and parallel flow velocity at $r=30 \mathrm{~mm}$ (b).
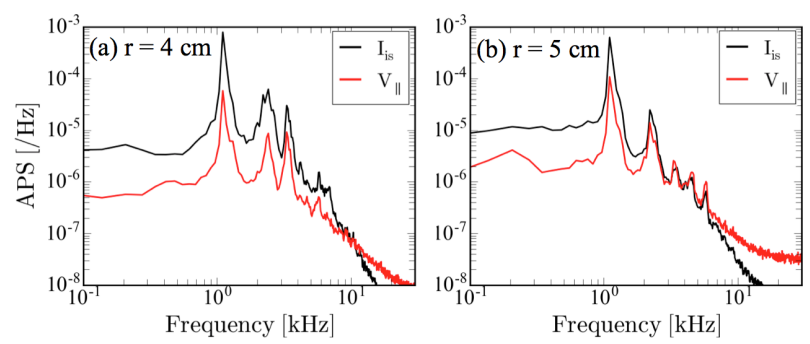

Fig. 2 Auto-power spectra for normalized ion saturation current and parallel flow velocity at $r=40 \mathrm{~mm}$ (a) and $50 \mathrm{~mm}$ (b). higher phase values. Then these radii might be favourable for D'AM generation at frequencies higher than $3.5 \mathrm{kHz}$.

We use the critical shear velocity value to verify this last point. It is given by $v_{\| c}^{\prime}=\left(c_{s} / L_{n}\right) /\left(1+\rho_{s}^{2} k_{\perp}^{2}\right)$ [4]. We use $\rho_{s}^{2} k_{\perp}^{2}=1$. The radial profile of $v_{\|}^{\prime} / v_{\| c}^{\prime}$ ratio is shown in Fig. 3 and demonstrate clearly that D'AMs can arise at $r=30 \mathrm{~mm}$, but not at higher radii.

Extensive study was realized at every radius. A global summary of the discrimination study based on power level is shown in Fig. 4. The red area is favourable to DW generation, while the blue area is favourable to D'AM generation. DW can propagate at every radii. However, higher frequencies exhibit clearly a difference of behaviour depending on the radius: at $30 \mathrm{~mm}$, D'AM can arise, especially at $4.5 \mathrm{kHz}$. On the other hand, radius $r=40 \mathrm{~mm}$ is unfavourable to such generation, and it is known to be the best radius for DW observation in PANTA [9]. Finally, higher radii are not favourable to DW generation, especially at $r=50 \mathrm{~mm}$. Radial mapping of auto-power spectrum for $v_{\|}$showed corresponding peaks at this position, matching frequencies of peaks at $r=30 \mathrm{~mm}$ : this might be due to coupling for eigenfunction, without D'AM generation (as proved with Fig. 3). This is outside the scope of the paper and could be further investigated in the future.

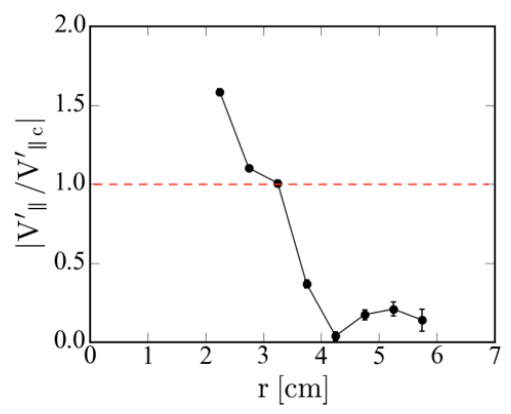

Fig. 3 Ratio of the parallel velocity to the critical shear required for the onset of D'Angelo mode. The red dotted line is marginal state: above it, D'Angelo modes are unstable.

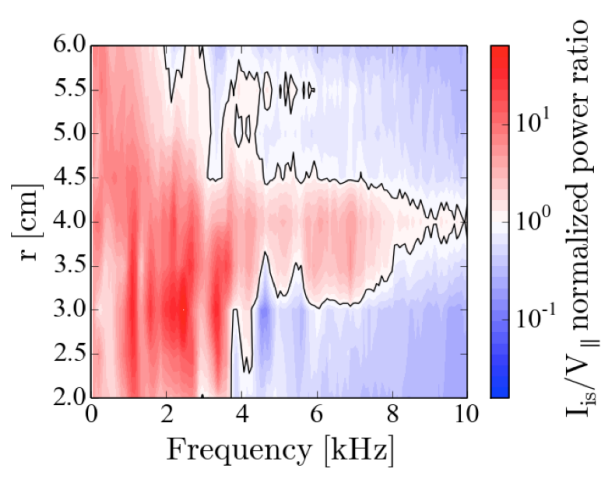

Fig. 4 Radial mapping of auto-power spectra ratio between normalized ion saturation current and parallel flow velocity. The black line exhibits the iso-contour of value 1 . 
To conclude, the coexistence of DW and D'AM fluctuations were investigated on PANTA. The ratio of normalized amplitudes of $v_{\|}$fluctuation and electron density fluctuation is used to discriminate the two phenomena. The study indicates that they coexist in the plasma, but the dominant amplitude of these fluctuations is located at different radial positions. Furthermore, radial map of power spectra for normalized $v_{\|}$suggests the presence of coupling for eigenfunction at $4.5 \mathrm{kHz}$ between $r=30 \mathrm{~mm}$ and $r=50 \mathrm{~mm}$. More detailed studies, including investigation of possible radial eigenfunction and spectrum form, will be pursued in the future.

We would like to acknowledge useful discussions with Mr. T. Mitsuzono, Dr. K. Yamasaki. This work was also partially supported by Grants-in-Aid for Scientific Research of JSPS, Japan (JP15H02155, JP15H02335 and JP16H02442), the collaboration programs of RIAM, Kyushu University, by Asada Science Foundation, by the
Friendship scholarship of Kyushu University, and by the financial aid from EPFL.

[1] B.B. Kadomtsev, Plasma Turbulence (Academic, New York, 1965).

[2] H. Hasegawa et al., Nature 430, 755 (2004).

[3] T. Kaneko, H. Tsunoyama and R. Hatakeyama, Phys. Rev. Lett. 90, 125001 (2003).

[4] S. Inagaki et al., Sci. Rep. 6, 22189 (2016).

[5] N. D’Angelo, Phys. Fluids 8, 1748 (1965).

[6] Y. Kosuga, S.-I. Itoh and K. Itoh, Plasma Fusion Res. 10, 3401024 (2015).

[7] W.E. Amatucci, J. Geophys. Res. 104, 14481 (1999).

[8] M. Sasaki et al., Japanese Physical Society Autumn Meeting 2016, 15pKA10.

[9] T. Yamada et al., Nature Phys. 4, 721 (2008).

[10] S. Oldenbürger et al., Plasma Fusion Res. 7, 2401146 (2012). 